\title{
Cathepsin S Inhibition Attenuates Neuropathic Pain and Microglial Response Associated with Spinal Cord Injury
}

\author{
Anna K. Clark, Fabien Marchand, Marta D’ Auria, Meirion Davies, John Grist, Marzia Malcangio* \\ and Stephen B. McMahon
}

Wolfson Centre for Age-Related Diseases, Kings College London, London, UK

\begin{abstract}
Loss of function is usually considered the major consequence of spinal cord injury (SCI). However, chronic pain severely compromises the quality of life of many SCI patients. Recently, microglial cells in enhanced response states have been proposed to contribute to chronic pain following SCI. Here we report that following contusion injury, the microglial cysteine protease cathepsin S (CatS) is critical for the maintenance of SCI-induced neuropathic pain and spinal microglial response. Following SCI, significant mechanical and thermal hypersensitivity developed in both hind-paws. Prolonged intrathecal administration of the CatS inhibitor LHVS (morpholinurea-leucine-homophenylalanine- vinyl sulfone-phenyl), commencing day 26 post-SCI, resulted in significant attenuation of established mechanical and thermal pain behaviours compared to vehicle. This attenuation was evident as early as $24 \mathrm{hrs}$ following treatment initiation, and was maintained throughout the 7 day duration of drug administration. In addition, following the 7 day treatment period LHVS significantly attenuated the SCI-induced response of microglial in the lumbar dorsal horn of the spinal cord. We suggest that following SCI, CatS expressed by spinal microglia, is critical for the maintenance of below the level pain induced by contusion injury and suggest that CatS inhibition constitutes a novel therapeutic approach for the treatment of chronic pain associated with SCI.
\end{abstract}

Keywords: Protease, microglia, hyperalgesia, chronic pain.

\section{INTRODUCTION}

Spinal cord injury (SCI) is a debilitating event leading to profound loss of motor function below the level of the lesion. Chronic central pain is a common occurrence, severely compromising the quality of life of many SCI patients. Studies indicate a prevalence of $65-85 \%$ of patients developing clinically significant pain following SCI [1], which is often refractory to current pharmacological treatments [2,3]. Despite the prevalence and severity of post-SCI pain, the mechanisms associated with this type of neuropathic pain have only been investigated relatively recently.

A major conceptual advance has been the recognition of the importance of neuroimmune interactions in the modulation of altered nociceptive processing in chronic pain states. Microglia respond quickly to injury, up-regulating cell surface proteins and increasing their synthesis and release of inflammatory mediators that can sensitize neurons, thereby establishing a positive feedback which contributes to chronic pain following peripheral nerve injuries $[4,5]$. Thus, following nerve injury microglial cells enter a pain-related enhanced response state [5]. In particular, the microglial cysteine protease Cathepsin $\mathrm{S}$ (CatS) is critical for neuropathic pain following peripheral nerve injury [6].

*Address correspondence to this author at the Wolfson Centre for AgeRelated Diseases, Kings College London, Wolfson Wing, Hodgkin Building, Guys Campus, London, SE1 1UL, UK; Tel: 02078486092; Fax: 02078486165; E-mail: marzia.malcangio@kcl.ac.uk
Spinal microglial activation has also been observed following SCI, both in human patients [7] and following experimental SCI models [8]. Recent evidence suggests that this microglial response in the lumbar dorsal horn contributes to the maintenance of below the level pain associated with SCI [9-12], as well as its initiation [13]. Following SCI, microglia contribute to enhanced neuronal hyperexcitability associated with pain behaviours [9, 11], and several mechanisms of microglial-neuronal communication have been proposed to mediate this phenomenon [8]. In particular, enhanced expression of pro-inflammatory cytokines that contribute to neuropathic pain following peripheral nerve injuries, contribute to below the level pain following SCI [14]. However, greater understanding of the mechanisms of post-SCI injury pain may reveal novel therapeutic approaches for the treatment of chronic pain.

In this study, we examine the contribution of microglial CatS, a key player in microglia-neuronal communication following peripheral nerve injury $[6,15]$, to the maintenance of below the level neuropathic pain associated with SCI.

\section{MATERIALS AND METHODS}

\section{Animals}

All experiments were carried out using adult male Wistar rats (220-250 g, Harlan, UK) housed in standard laboratory conditions with free access to food and water. All experiments were undertaken with approval of the United Kingdom Home Office. Experimental study groups were randomized and blinded. In total 18 rats were used for this study. 


\section{Surgical Procedures}

\section{Contusion Injury}

Adult male rats were anaesthetised with an intraperitoneal injection of medetomidine $(0.25 \mathrm{mg} / \mathrm{kg})$ and ketamine $(60 \mathrm{mg} / \mathrm{kg})$, and body temperature was monitored with a rectal thermometer and maintained at $37^{\circ} \mathrm{C}$. SCI was produced at spinal segment T10 using the Infinite Horizon Impactor device (The Ohio State University, USA). A laminectomy was performed at T10 with the vertebral column stabilized at T8 and T11 using angled clamps. The impactor tip ( $2 \mathrm{~mm}$ diameter) was positioned over the exposed dura and triggered to deliver a contusion injury with a force of 200 Kdynes. The wound was rinsed with saline, and the overlying muscles and skin were closed in layers with 4-0 silk sutures. Animals were allowed to recover on a $30^{\circ} \mathrm{C}$ heating pad. Postoperative treatment included saline (2.0 cc, s.c., twice daily) to prevent dehydration. Bladders were manually expressed twice daily until reflex bladder emptying returned.

\section{Intrathecal Pumps}

Intrathecal cannulas were implanted directly following contusion injury (at day 0). Under anaesthesia a midline incision was made over the midthoracic spinal cord. Muscles were separated from bone by blunt dissection, and a small laminectomy was made at the sixth or seventh thoracic vertebra. An intrathecal cannula $(0.30 \mathrm{~mm}$ I.D., $0.63 \mathrm{~mm}$ O.D.) was inserted under the dura mater such that the tip rested at the lumbar enlargement and the opposite end sealed with glue. Immediately following behavioural testing on day 26 post-SCI the end of previously implanted cannulae tubing was attached a subcutaneous osmotic mini-pump (Alzet model 2001) containing the irreversible CatS inhibitor LHVS (morpholinurea-leucine-homophenylalanine- vinyl sulfone-phenyl; NeoMPS Inc, San Diego, CA, USA) or vehicle ( $20 \%$ cremophor EL/saline; Sigma, UK). Vehicle or LHVS (30nmol/day) were administered intrathecally for 7 days (beginning day 26 post-SCI). Proper placement of the cannulae was determined in each animal at the end of the experiment.

\section{Behavioural Testing}

\section{Mechanical Allodvnia}

Mechanical withdrawal thresholds were assessed by measuring paw withdrawal threshold (PWT) using a Dynamic Plantar Anesthesiometer (Ugo Basile, Italy). In brief, each animal was placed in a clear acrylic cubicle $(22 \mathrm{x}$ $16.5 \times 14 \mathrm{~cm})$ on top of a metal grid in a temperature controlled room $\left(\sim 22^{\circ} \mathrm{C}\right)$ and allowed to acclimatise for 15 minutes before testing. The stimulus was applied via an actuator filament $(0.5 \mathrm{~mm}$ diameter) which under computer control applied a linearly increasing force ramp $(2.5 \mathrm{~g} / \mathrm{sec})$ to the plantar surface of the hind paw. The force necessary to elicit a paw withdrawal was recorded. A cut-off of $50 \mathrm{~g}$ was imposed to prevent any tissue damage. The withdrawal threshold of each hindpaw was calculated as the average of three consecutive tests with at least 5 minutes between each test. Measurements were taken on three separate days prior to surgery, on two separate days following surgery and then throughout the 7 day treatment period. Following SCI behavioural tests were only conducted once full motor impairment of the hind-limbs had resolved in order for reliable withdrawal thresholds to be recorded.

\section{Thermal Hyperalgesia}

Using the method described by Hargreaves et al. [16]. In brief, each animal was placed in a clear acrylic cubicle $(22 \mathrm{x}$ $16.5 \times 14 \mathrm{~cm}$ ) on top of a glass floor in a temperature controlled room $\left(\sim 22^{\circ} \mathrm{C}\right)$ and allowed to acclimatise for 15 minutes before testing. The withdrawal latencies of each hindpaw were averaged over three consecutive tests, at least 5 minutes apart, in response to the thermal challenge from a calibrated (output of $190 \mathrm{~mW} / \mathrm{cm}^{2}$ ) radiant heat light source. A cut-off of 20 seconds was imposed to prevent tissue damage. Measurements were taken on three separate days prior to surgery, on two separate days following surgery and then throughout the 7 day treatment period.

\section{Immunohistochemistry}

At the completion of behavioural experiments, animals were under pentobarbital anaesthesia, transcardially perfused with $0.9 \%$ saline solution followed by $4 \%$ paraformaldehyde in $0.1 \mathrm{M}$ phosphate buffer. The lumbar spinal cord was excised, post-fixed for $4 \mathrm{hr}$ in the perfusion fixative, then cryoprotected in $20 \%$ sucrose in $0.1 \mathrm{M}$ phosphate buffer $\left(72 \mathrm{hr}\right.$ at $\left.4^{\circ} \mathrm{C}\right)$ and frozen in O.C.T embedding compound (VWR, UK). Transverse sections $(20 \mu \mathrm{m})$ were cryostat cut and thaw-mounted onto glass slides. Slides were incubated overnight with primary antibody solution for Iba-1 (rabbit anti-ionized calcium binding adapter molecule 1, 1:1000, Wako Chemicals, Germany) or OX42 (mouse anti-Cd11b, $1: 100$, Serotec, UK) followed by secondary antibody solution (IgG-conjugated Alexa Fluor 488 ${ }^{\mathrm{TM}}$, Molecular Probes, OR, USA). Slides were cover-slipped with Vectashield mounting medium (Vector Laboratories, CA, USA) and visualised under a Zeiss Axioplan 2 fluorescent microscope.

\section{Quantification and Statistics}

Quantitative assessment of Iba-1 and OX42 immunostaining was carried out by counting the number of positive profiles within a fixed area of the dorsal horn $[6,15$, 17, 18]. A box measuring $10^{4} \mu \mathrm{m}^{2}$ was placed onto areas of the lateral, central and medial dorsal horn and the number of profiles positive for each marker counted within this area. These measurement protocols were carried out on three L5 spinal sections from each animal. For the analysis of behavioural data two-way repeated measures of ANOVA followed by Tukey's post-hoc test was used. For immunohistochemical data one-way repeated measures of ANOVA followed by Tukey's post-hoc test was used. $\mathrm{P}<0.05$ was set as the level of statistical significance.

\section{RESULTS}

\section{Inhibition of CatS Attenuates Established Neuropathic Pain Behaviours Following SCI}

Despite the now established role of microglial in enhanced response states to neuropathic pain following peripheral nerve injury $[4,5]$, spinal microglia have only recently been reported to contribute to chronic pain in rodent models of SCI [8]. We have previously reported that 
inhibition of the microglial enzyme CatS is able to reverse established pain behaviours following peripheral nerve injury [6]. We therefore examined the contribution of spinal CatS to neuropathic pain behaviours and microglial response associated with SCI.

Contusion of the spinal cord at T10 resulted in mechanical allodynia (Fig. 1A), indicated by a significant reduction in mechanical withdrawal thresholds of both hind paws 26 days following SCI compared to baseline PWT. Both hind-paws showed similar levels of mechanical allodynia. Animals also demonstrated significant thermal hyperalgesia on day 26 following SCI (Fig. 1B). Continuous intrathecal administration of the CatS inhibitor LHVS, from day 26-33 following SCI, significantly attenuated both mechanical and thermal hypersensitivity commencing on day 27, compared to vehicle (Fig. 1A-B). Gross observation of motor behaviour did not reveal difference in ambulation between LHVS and vehicle treated groups, however animals had recovered from SCI-induced motor impairment by the time treatments were started. These data suggest a role for spinal CatS in the maintenance of SCI induced neuropathic pain behaviours.

\section{Microglial Response Following SCI is Attenuated by CatS Inhibition}

We have previously reported that inhibition of CatS following peripheral nerve injury reduces the number of microglial cells in enhanced response states in the dorsal horn of the spinal cord [6]. We therefore examined the effect of CatS inhibition on the microglial response following SCI using immunohistochemistry. Thirty-three days following SCI a significant enhancement in microglial response was observed in animals receiving vehicle treatment compared to intact animals. Quantification of immunohistochemical markers revealed that SCI significantly increased numbers of Iba-1 (Fig. 2A-B) and OX42 (Fig. 2C) positive microglia in both dorsal horns. Animals receiving LHVS treatment following SCI also exhibited enhanced microglial response compared to intact controls (Fig. 2). However, intrathecal LHVS significantly reduced the number of microglia expressing both Iba-1 (Fig. 2A-B) and OX42 (Fig. 2C) in the lumbar dorsal horn compared to vehicle treated animals. These data suggest that the anti-hyperalgesic effects of LHVS following SCI are as a result of a reduction in microglial response in the dorsal horn of the spinal cord.

\section{DISCUSSION}

Here we demonstrate that the microglial enzyme CatS is critical for SCI induced neuropathic pain behaviour and microglial response. Specifically, following contusion SCI both mechanical and thermal hypersensitivity develop in the hind-paws and enhanced microglial response is observed in the dorsal horn of the lumbar spinal cord. Chronic intrathecal treatment with a CatS inhibitor is able to attenuate established pain behaviours and reduce spinal microglial response, suggesting that CatS is vital for the maintenance of microglial driven neuropathic pain following SCI.

Chronic neuropathic pain is a major complication of SCI. Several established animal models of SCI manifest
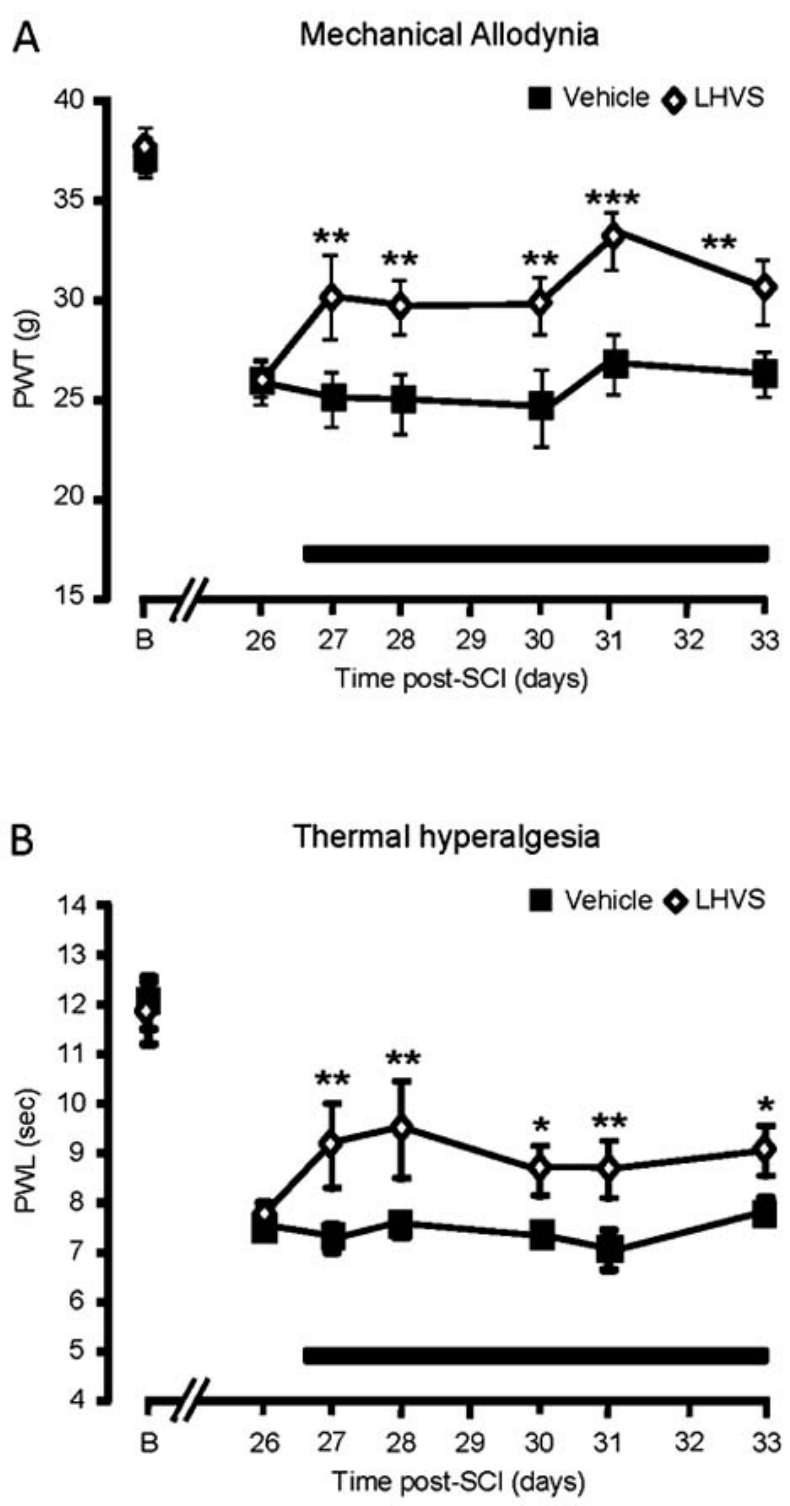

Fig. (1). LHVS attenuates neuropathic pain behaviour following spinal cord injury. Rats develop significant mechanical allodynia (A) and thermal hyperalgesia (B) following spinal cord contusion which is partially reversed by continuous intrathecal infusion of LHVS. $\mathrm{n}=9$ rats per group. $* * * \mathrm{P}<0.001, * * \mathrm{P}<0.01, * \mathrm{P}>0.05$ verses vehicle treated group, Two Way-ANOVA, post-hoc Tukey.

behavioural patterns which are considered representative of the main features of human SCI pain. The contusion model of SCI is believed to be the most clinically relevant, closely paralleling the injury profile of many patients [19]. The chronic pain associated with this model of SCI has been extensively characterized, with animals exhibiting thermal and mechanical allodynia at the level of injury (at-the-level pain), as well as in both forepaws (above-the-level pain) and hind-paws (below-the-level pain) [20]. Following contusion of the spinal cord at T10 we observed the development of below the level pain. Consistent with previous studies $[9,11$, 14, 20-23], animals exhibited both thermal and mechanical pain behaviours in both hind-paws following SCI. 

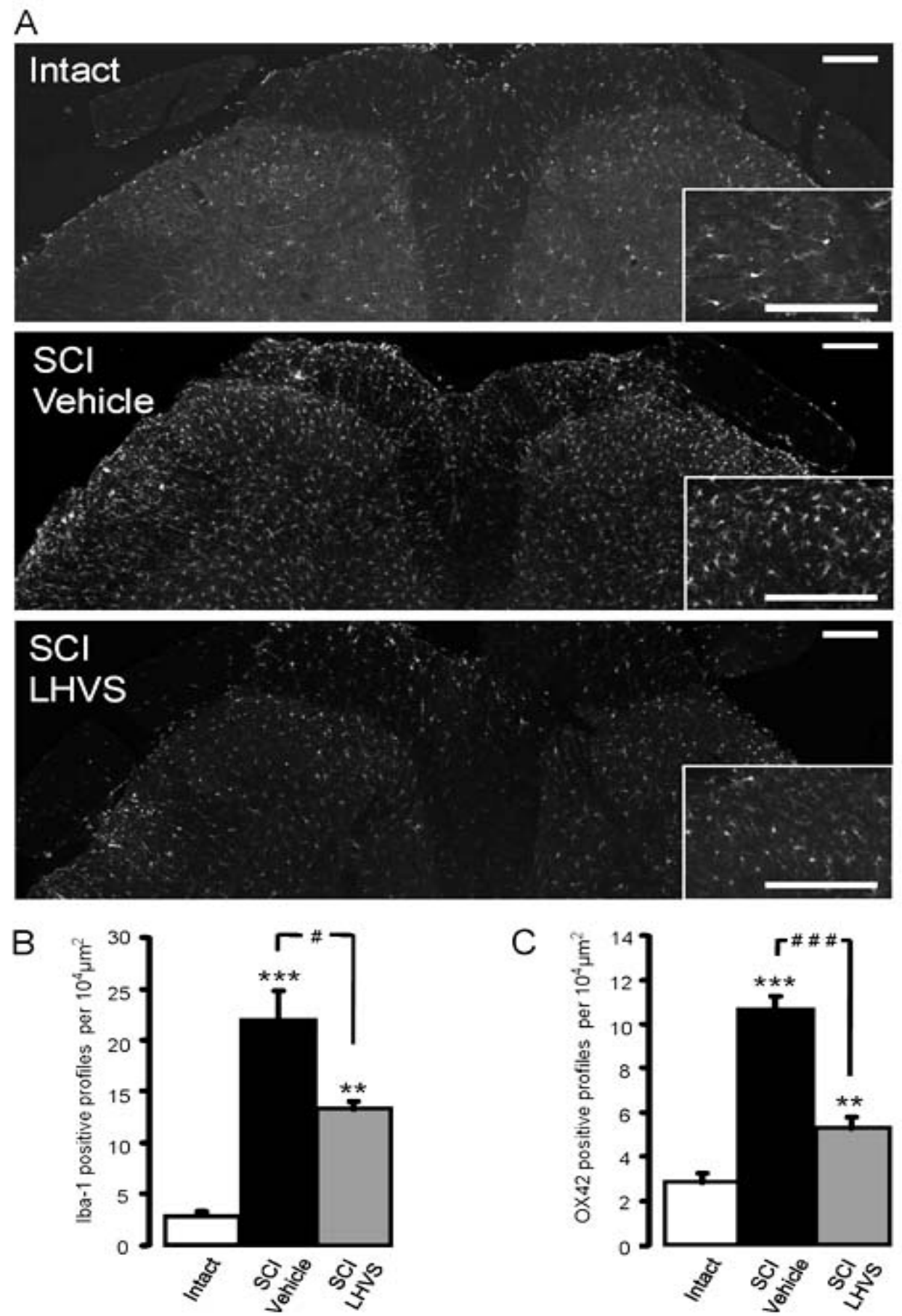

Fig. (2). Spinal cord injury increases lumbar microglial response, which is attenuated by LHVS. A: Following spinal cord injury increased numbers of Iba-1 positive microglia are observed in the lumbar spinal cord. This increase in cell number is partially reversed by LHVS treatment. B: Quantification of Iba-1 positive microglia. $\mathrm{n}=4-5$ per rats group. ${ }^{*} * * \mathrm{P}>0.001, * * \mathrm{P}>0.01$ verses naive, \#P>0.05 verses vehicle treated group. C: Quantification of $\mathrm{OX} 42$ positive microglia. $\mathrm{n}=4-5$ per rats group. ${ }^{* * *} \mathrm{P}>0.001, * * \mathrm{P}>0.01$ verse naive, \#\#\#P>0.001 verses vehicle treated group.

Substantial evidence now demonstrates that non-neuronal cells critically contribute to the full development of neuropathic pain in a variety of animal models $[4,5]$. Spinal microglia respond quickly to injury, up-regulating cell surface proteins and increasing their synthesis and release of inflammatory mediators, including cytokines and proteases that can modulate neuronal sensitivity thereby contributing to increased nociception following injury [4]. In particular, the microglial protease CatS and the neuronal chemokine Fractalkine (FKN) represent a key neuron-microglia signaling pair during neuropathic pain $[6,15,24]$. CatS is upregulated in microglial cells following nerve injury $[6$, 
25], and CatS inhibitors reverse neuropathic pain behaviours $[6,26,27]$. Following microglia activation CatS is released into the extracellular environment [28], where it exerts its pro-nociceptive effects via cleavage of membrane-bound FKN $[6,15]$. Soluble FKN is then able to further amplify the pain-related enhanced response state of microglial via activation of the CX3CR1 receptor. Hence, the inhibition of microglial targets such as CatS [6, 26, 27] and CX3CR1 [29] can reduce hypersensitivity in chronic pain states.

More recently spinal microglial in enhanced response states have also been proposed to play a role in chronic SCI pain [8]. Here we demonstrate enhanced microglial response in the lumbar spinal cord following T10 contusion injury. Indeed, enhanced microglial response in the lumbar spinal cord, many spinal segments below the SCI site, has been reported following both contusion [9, 11, 14, 23] and hemisection injury $[10,13,30]$. In addition, microglial inhibitors such as minocycline are able to delay the development of below the level SCI induced pain behaviours [13], as well as reverse established neuropathic pain [9].

Here we provide evidence that chronic intrathecal administration of the irreversible CatS inhibitor, LHVS, significantly reverses established mechanical and thermal hypersensitivity following SCI. In addition, enhanced nociceptive thresholds were found to correlate with decreased microglial cell number and response state in the lumbar dorsal horn. These data suggest that the anti-allodynic effects of CatS inhibition are mediated via an attenuation of microglia activity. Indeed, we have previously reported that following peripheral nerve injury CatS inhibition is antiallodynic via a reduction in spinal microglial response [6]. In addition, LHVS is able to diminish both p38 MAPK phosphorylation and secretion of Interleukin- $1 \beta$ from spinal microglia following lipopolysaccharide stimulation [15], suggesting that CatS inhibition reduces the overall proinflammatory nature of microglial cells. Therefore, the effects of such pharmacological agents may be two fold, acting directly to dampen the pro-inflammatory nature of spinal microglia by decreasing the synthesis of microglial mediators. Indirectly, a reduction in such inflammatory agents may act to reduce the hyperexcitability of spinal neurons observed following SCI $[9,11]$, leading to an overall attenuation of chronic pain. Indeed, many inflammatory mediators found within the CNS are able to enhance neuronal excitability $[12,31]$.

Our study reinforces the role of microglial in chronic pain following SCI and proposes CatS as a new signaling candidate contributing to SCI pain. However, the factors that initiate changes in microglial response state as well as the mediators that are thought to contribute to the production of pain still require extensive study. Several microglia-neuron signaling pathways have been suggested to contribute to chronic pain. We have previously demonstrated that following peripheral nerve injury CatS mediates its pronociceptive effects via cleavage of the neuronal chemokine FKN [15]. We suggest that this microglial-neuronal signaling pathway may also contribute to SCI-induced neuropathic pain, as CatS inhibition effectively attenuates both pain behaviours and microglial response following SCI. A second suggested microglia-neuron signaling pair may comprise microglial Extracellular Signal-Related Kinase
(ERK) and neuronal Prostaglandin E2 [11]. Following SCI, phosphorylation of mitogen activated protein kinases (MAPK) including p38 [9, 10] and ERK [11] is observed in spinal microglia. ERK phosphorylation may then lead to enhanced secretion of Prostaglandin E2 which acts to enhance neuronal excitability [11]. Thirdly, the chemokine CCL21 has been proposed to act as a remote activator of thalamic microglia following SCI [12]. However, further studies are needed to clarify the role of particular microglia mediators in central injury and their contribution to chronic pain.

In conclusion, our results reinforce the role of microglia in SCI-induced pain and establish a new role for the microglial protease CatS in the maintenance of neuropathic hypersensitivity following contusion SCI. We suggest that CatS inhibition constitutes a novel therapeutic approach for the treatment of chronic pain following SCI.

\section{ACKNOWLEDGEMENTS}

This work was supported by the Wellcome Trust (AKC, $\mathrm{MM}$ ) and the ISRT (FM, SBM).

\section{REFERENCE}

[1] Siddall PJ, McClelland JM, Rutkowski SB, Cousins MJ. A longitudinal study of the prevalence and characteristics of pain in the first 5 years following spinal cord injury. Pain 2003; 103: 24957.

[2] Siddall PJ. Management of neuropathic pain following spinal cord injury: now and in the future. Spinal Cord 2009; 47(5):352-9.

[3] Bastrup C, Finnerup NB: Pharmacological management of neuropathic pain following spinal cord injury. CNS Drugs 2008; 22: 455-75.

[4] Milligan ED, Watkins LR. Pathological and protective roles of glia in chronic pain. Nat Rev Neurosci 2009; 10: 23-36.

[5] McMahon SB, Malcangio M. Current challenges in glia-pain biology. Neuron 2009; 64: 46-54.

[6] Clark AK, Yip PK, Grist J, et al. Inhibition of spinal microglial cathepsin S for the reversal of neuropathic pain. Proc Natl Acad Sci USA 2007; 104: 10655-60.

[7] Chang HT. Subacute human spinal cord contusion: few lymphocytes and many macrophages. Spinal Cord 2007; 45: 174182.

[8] Hulsebosch CE, Hains BC, Crown ED, Carlton SM. Mechanisms of chronic central neuropathic pain after spinal cord injury. Brain Res Rev 2009; 60: 202-213.

[9] Hains BC, Waxman SG. Activated microglia contribute to the maintenance of chronic pain after spinal cord injury. J Neurosci 2006; 26: 4308-17.

[10] Peng XM, Zhou ZG, Glorioso JC, Fink DJ, Mata M. Tumor necrosis factor-alpha contributes to below-level neuropathic pain after spinal cord injury. Ann Neurol 2006; 59: 843-51.

[11] Zhao P, Waxman SG, Hains BC. Extracellular signal-regulated kinase-regulated microglia-neuron signaling by prostaglandin e2 contributes to pain after spinal cord injury. J Neurosci 2007; 27: 2357-68.

[12] Zhao P, Waxman SG, Hains BC. Modulation of thalamic nociceptive processing after spinal cord injury through remote activation of thalamic microglia by cysteine cysteine chemokine ligand 21. J Neurosci 2007; 27: 8893-8902.

[13] Marchand F, Tsantoulas C, Singh D, et al. Effects of etanercept and minocycline in a rat model of spinal cord injury. Eur J Pain 2009; 13: 673-81.

[14] Detloff MR, Fisher LC, McGaughy V, Longbrake EE, Popovich PG, Basso DM. Remote activation of microglia and proinflammatory cytokines predict the onset and severity of belowlevel neuropathic pain after spinal cord injury in rats. Exp Neurol 2008; 212: 337-47.

[15] Clark AK, Yip PK, Malcangio M. The liberation of fractalkine in the dorsal horn requires microglial cathepsin S. J Neurosci 2009; 29: 6945-54. 
[16] Hargreaves K, Dubner R, Brown F, Flores C, Joris J. A new and sensitive method for measuring thermal nociception in cutaneous hyperalgesia. Pain 1988; 32: 77-88.

[17] Clark AK, Gentry C, Bradbury EJ, McMahon SB, Malcangio M. Role of spinal microglia in rat models of peripheral nerve injury and inflammation. Eur J Pain 2007; 11: 223-30.

[18] Clark AK, Staniland AA, Marchand F, Kaan TKY, McMahon SB, Malcangio M. P2X7-Dependent release of interleukin-1\{beta $\}$ and nociception in the spinal cord following lipopolysaccharide. J Neurosci 2010; 30: 573-82.

[19] Bunge RP, Puckett WR, Becerra JL, Marcillo A, Quencer RM. Observations on the pathology of human spinal cord injury. A review and classification of 22 new cases with details from a case of chronic cord compression with extensive focal demyelination. Adv Neurol 1993; 59: 75-89.

[20] Hulsebosch CE, Xu GY, Perez-Polo JR, Westlund KN, Taylor CP, McAdoo DJ. Rodent model of chronic central pain after spinal cord contusion injury and effects of gabapentin. J Neurotrauma 2000; 17: 1205-17.

[21] Mills CD, Hains BC, Johnson KM, Hulsebosch CE. Strain and model differences in behavioral outcomes after spinal cord injury in rat. J Neurotrauma 2001; 18: 743-56.

[22] Hains BC, Yucra JA, Hulsebosch CE. Reduction of pathological and behavioral deficits following spinal cord contusion injury with the selective cyclooxygenase-2 inhibitor NS-398. J Neurotrauma 2001; 18: 409-23.

[23] Hains BC, Klein JP, Saab CY, Craner MJ, Black JA, Waxman SG, Upregulation of sodium channel Nav1.3 and functional involvement in neuronal hyperexcitability associated with central neuropathic pain after spinal cord injury. J Neurosci 2003; 23: 8881-92.

[24] Clark AK, Malcangio M. The Cathepsin S/Fractalkine pair: new players in spinal cord neuropathic pain mechanisms. In: Malcangio M, Ed. Synaptic Plasticity in Pain. New York: Springer 2009; pp. 455-71.

[25] Leichsenring A, Backer I, Wendt W, et al. Differential expression of Cathepsin $\mathrm{S}$ and $\mathrm{X}$ in the spinal cord of a rat neuropathic pain model. BMC Neurosci 2008; 9: 80.

[26] Irie O, Kosaka T, Ehara T, et al. Discovery of orally bioavailable cathepsin S inhibitors for the reversal of neuropathic pain. J Med Chem 2008; 51: 5502-5.

[27] Barclay J, Clark AK, Ganju P, et al. Role of the cysteine protease cathepsin S in neuropathic hyperalgesia. Pain 2007; 130: 225-34.

[28] Clark AK, Wodarski R, Guida F, Sasso O, Malcangio M. Cathepsin $\mathrm{S}$ release from primary cultured microglia is regulated by the $\mathrm{P} 2 \mathrm{X} 7$ receptor. Glia 2010; DOI: 10.1002/glia.21042.

[29] Staniland AA, Clark AK, Wodarski R, et al. Reduced inflammatory and neuropathic pain and decreased spinal microglial response in fractalkine receptor (CX3CR1) knockout mice. J Neurochem 2010; 14: 1143-57.

[30] Gwak YS, Crown ED, Unabia GC, Hulsebosch CE. Propentofylline attenuates allodynia, glial activation and modulates GABAergic tone after spinal cord injury in the rat. Pain 2008; 138: 410-22.

[31] Owolabi SA, Saab CY. Fractalkine and minocycline alter neuronal activity in the spinal cord dorsal horn. FEBS Lett 2006; 580: 430610

\section{(C) Clark et al.; Licensee Bentham Open.}

This is an open access article licensed under the terms of the Creative Commons Attribution Non-Commercial License (http://creativecommons.org/licenses/by-nc/3.0/) which permits unrestricted, non-commercial use, distribution and reproduction in any medium, provided the work is properly cited. 